\title{
Conduction mechanism of metal-free phthalocyanine single crystals as a function of temperature
}

\author{
AYONA SIMON and FRANCIS P XAVIER* \\ Physics Department, Loyola Institute of Frontier Energy (LIFE), Loyola College, Chennai \\ 600034. India \\ MS received 26 February 1996; revised 15 April 1997

\begin{abstract}
Metal-free phthalocyanine $\left(\mathrm{H}_{2} \mathrm{Pc}\right)$ single crystals grown by vacuum sublimation were investigated for their conductivity (both in dark and light). The investigations consisted of dark-and photo-current variations with (i) applied electric field and (ii) temperature. The applied electric field ranged from $0.8 \mathrm{kV} / \mathrm{cm}$ to $6 \mathrm{kV} / \mathrm{cm}$. The temperature range was from $300 \mathrm{~K}$ to around $570^{\circ} \mathrm{K}$. The crystals were found to be photoconductive. Based on activation energies calculated from photoconductivity due to temperature dependence, an energy level scheme for $\mathrm{H}_{2} \mathrm{Pc}$ single crystals is proposed. The model consists of two trapping levels within the forbidden gap- - one at $0.4 \mathrm{eV}$ below the conduction band edge from which electrons are thermally excited into the conduction band and the other acts as recombination centre at $0.3 \mathrm{eV}$ above the valence band edge. The band gap is calculated to be $1.4 \mathrm{eV}$. Comparative study of the proposed model with that of earlier investigations on the same crystals of the $\mathrm{H}_{2} \mathrm{Pc}$ is in good agreement, thereby indicating that $\mathrm{H}_{2} \mathrm{Pc}$ is thermally stable even at relatively higher temperature as semiconductor.
\end{abstract}

Keywords. Phthalocyanine: photoconductor; single crystals.

\section{Introduction}

In today's world of technology there seems to be competition between organic and inorganic semiconductors. A typical organic material is neither a simple covalently nor an ionically bonded crystal but a molecular crystal. And when they are further semiconducting these crystals are called 'organic semiconductors'. Organic semiconductors consist of regular array of closely spaced molecules. The intermolecular binding is the weak van der Waals force but the electrons within a molecule are tightly bound (Xavier 1993). Photoconductivity is an important feature of organic semiconductors. It is a process in which charge carriers are generated and transported under illumination. Phthalocyanine ( $\mathrm{Pc}$ ) is one of the most extensively studied organic semiconductors which find extensive applications as solar energy cells (Orti et al 1990) and in textile and paint industries as dyes (Orti et al 1988). It is further expected to serve as active material for molecular electronics such as chemical sensors (Minami et al 1987).

In the case of photoconductors it is essential to make sure that they are chemically and thermally stable over a period of time. In the present investigations Pc crystals which were grown a few years ago were investigated for temperature-dependence of conductivity at higher temperature range $\left(300^{\circ} \mathrm{K}-570^{\circ} \mathrm{K}\right)$ in order to establish whether they are stable as photoconductors over passage of time.

In organic semiconductors carrier generation and absorption are intra-molecular whereas transport of charge carriers is inter-molecular which is a solid-state property

*Address for communication. 
(Arulselvi and Xavier 1996). Hence electrical transport studies could be used to investigate the transport mechanism in organic semiconductors as in the case of conventional semiconductors.

\section{Sample preparation}

The Pc single crystals were prepared by Xavier and Goldsmith (1995) from the commercially available $\mathrm{H}_{2} \mathrm{Pc}$ powder $(99.9 \%$ pure-Fisher Scientific) by vacuum sublimation method. When a temperature of about $730^{\circ} \mathrm{K}$ was maintained at the powder sample, crystals in the form of whiskers were formed at a suitable region in the temperature gradient (about $610^{\circ} \mathrm{K}$ ) with dimensions of $15-20 \times 0.3$ $\times 0.14 \mathrm{~mm}$.

The sample crystals were fixed onto microscope slides with thin copper wires (of diameter $0.2 \mathrm{~mm}$ ) as electrodes (with electrode distance of about $1.3 \mathrm{~mm}$ ) and electrical connections were made in series with a de power supply and a picoammeter (Keithley

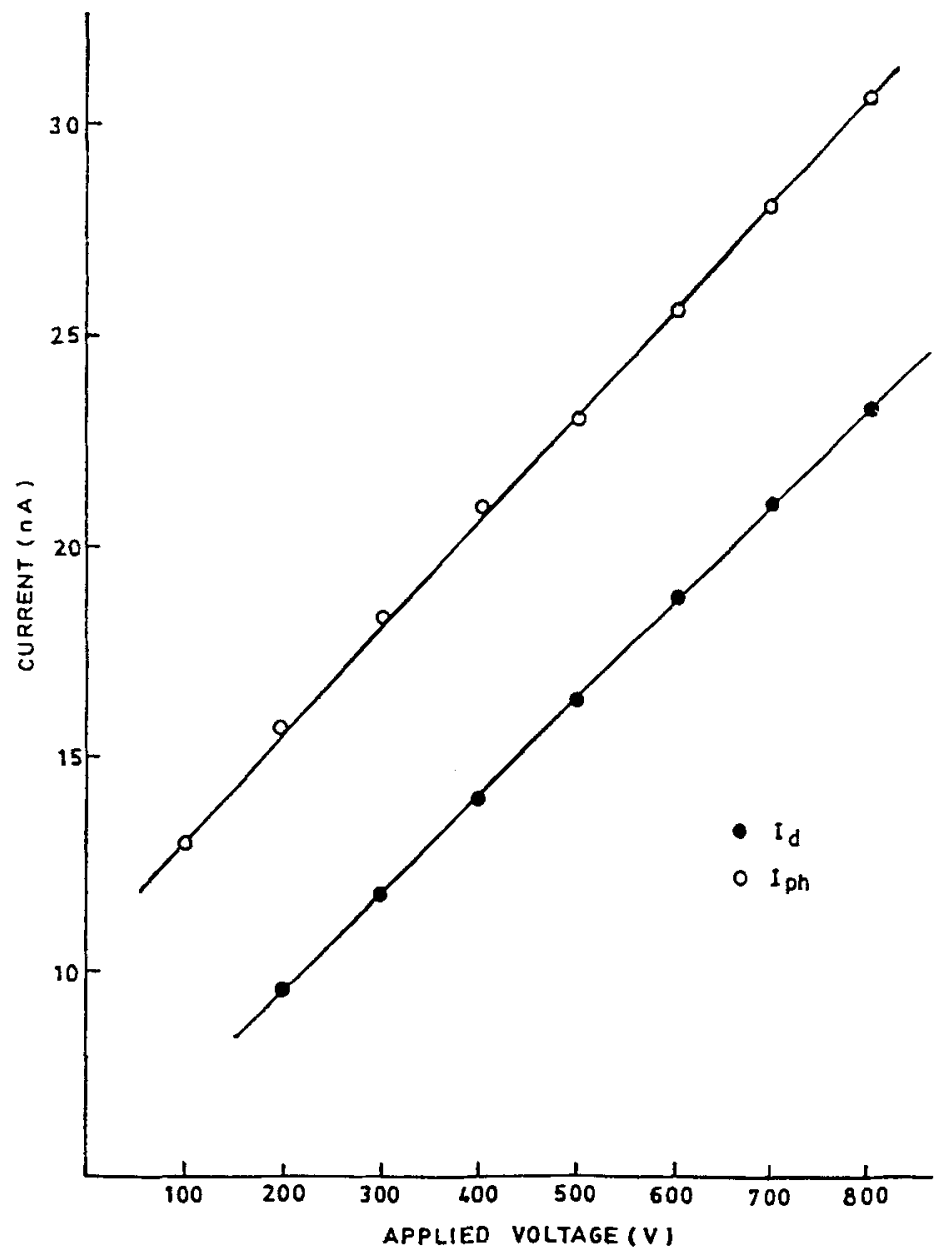

Figure 1. Current vs applied electric field. 
480). The dc input was increased in steps from $1.5 \mathrm{kV} / \mathrm{cm}$ to $6 \mathrm{kV} / \mathrm{cm}$ and the temperature range was increased up to $570^{\circ} \mathrm{K}$ from room temperature.

\section{Experimental}

\subsection{Current vs applied electric field}

Both dark $\left(I_{\mathrm{d}}\right)$ and photo $\left(I_{\mathrm{ph}}\right)$ currents were measured as a function of the applied field in order to establish the ohmicity of electrical contacts of the crystals. $I_{\mathrm{d}}$ as well as $I_{\mathrm{ph}}$ increased linearly with increased applied electric field along the crystal axis (b-axis) which indicated the ohmic contact of the electrodes (figure 1). The ratio of the photocurrent to dark current was about 1.5 and the resistivity of the $\mathrm{H}_{2}$ Pc crystal in the dark as well as in the light was of the order of $10^{8} \mathrm{ohm} \cdot \mathrm{cm}$.

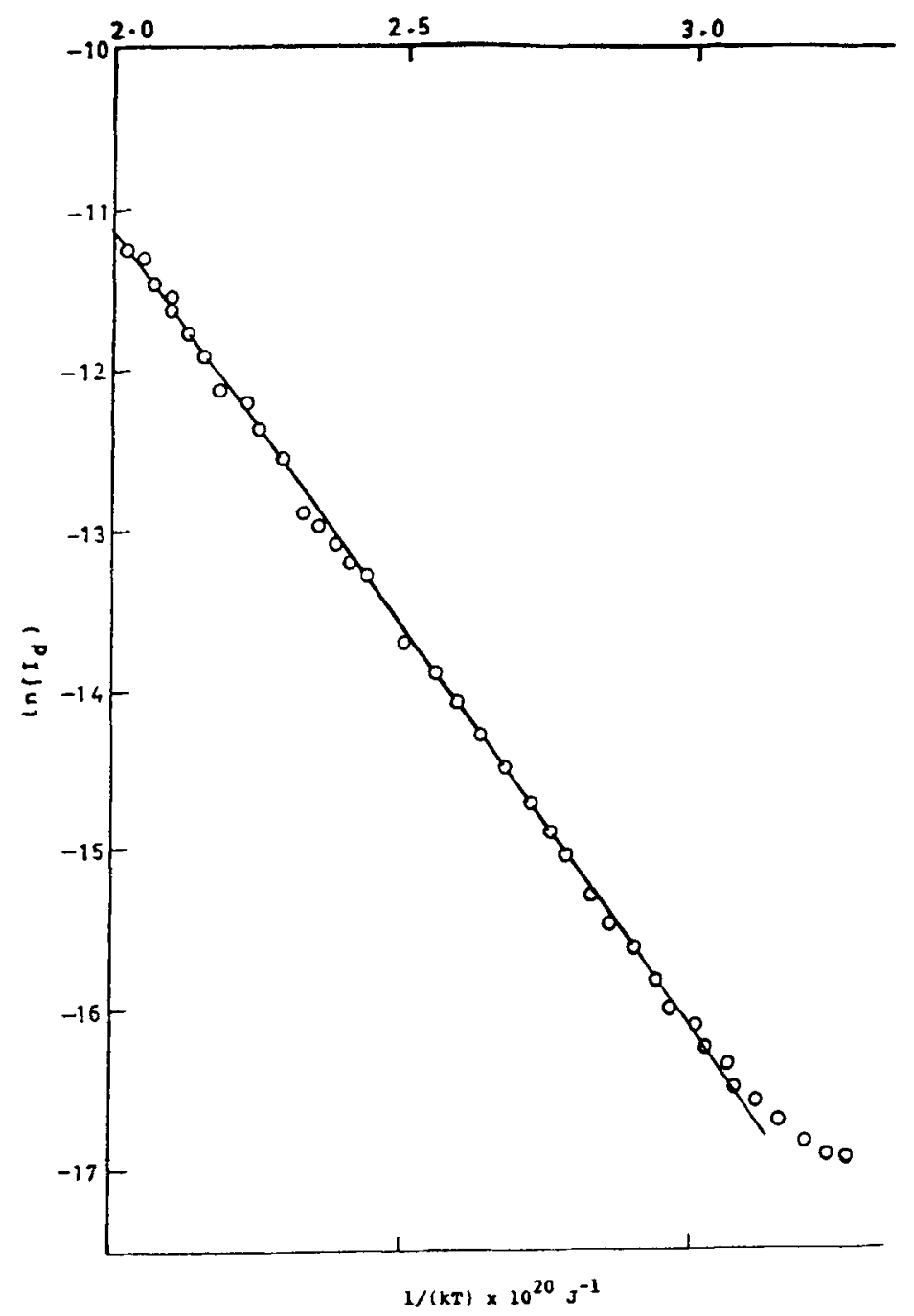

Figure 2. $\ln \left(I_{\mathrm{d}}\right)$ vs $(1 / T)$ of $\mathrm{H}_{2}$ Pc crystal. 


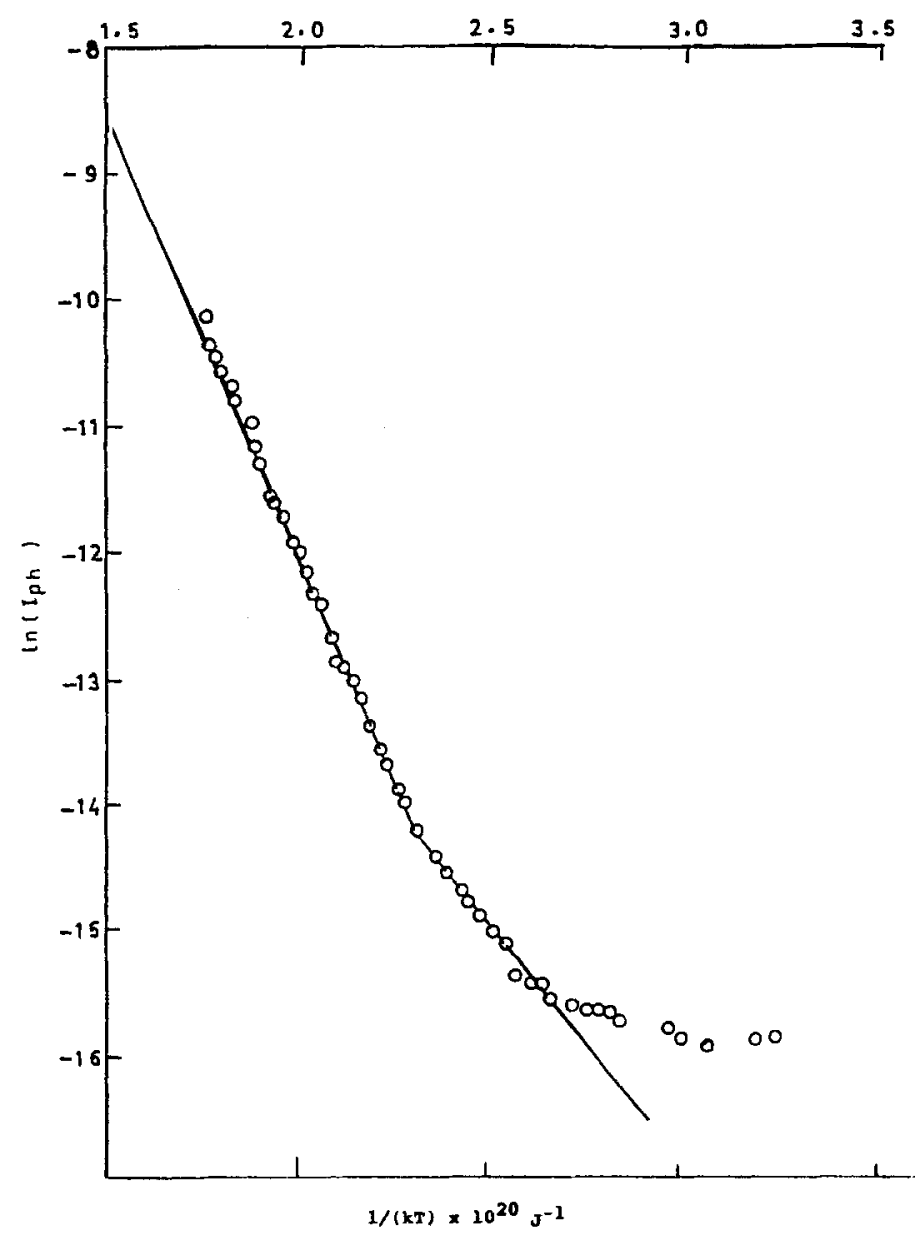

Figure 3. $\ln \left(I_{\mathrm{ph}}\right)$ vs $(1 / T)$ of $\mathrm{H}_{2} \mathrm{Pc}$ crystal.

\subsection{Dark current vs temperature}

The variation of $I_{\mathrm{d}}$ with temperature was measured for the temperature range between $300^{\circ} \mathrm{K}$ and $580^{\circ} \mathrm{K}$. It was found that the dark current, $I_{\mathrm{d}}$, increased exponentially with temperature, $T$. The dark-activation energy, $E$, of $\mathrm{H}_{2} \mathrm{Pc}$ single crystal was calculated as $0 \cdot 4 \mathrm{eV}$ using the relation

$$
I_{\mathrm{d}}=I_{0} \exp \{-E / k T\} \text {. }
$$

This is obtained as the slope of the plot $\ln \left(I_{\mathrm{d}}\right)$ vs $(1 / T)$ as shown in figure 2. By investigating the resistance variation, $R$, of the single crystals on increasing temperature, $T$ (plot of $\ln R$ vs $1 / T$ not shown), the band gap, $E_{\mathrm{g}}$, was calculated using the phenomenological relation (Simon and Andre 1985), due to inhomogeneity for most semiconductors (Boer 1990), given as

$$
R=R_{0} \exp \left\{E_{\mathrm{g}} / 2 k T\right\},
$$

to be $1.4 \mathrm{eV}$ from the activation energy $\left(E=E_{\mathrm{g}} / 2=0.7 \mathrm{eV}\right)$ determined from the slope of plot $\ln R$ vs $(1 / k T)$. 


\subsection{Photocurrent vs temperature}

The photocurrent, $I_{\mathrm{ph}}$, also increased exponentially with temperature, $T$. To calculate the photo activation energy, $E$, the relation

$$
I_{\mathrm{ph}}=I_{0} \exp \{-E / k T\},
$$

was used. And from the plot of $\ln \left(I_{\mathrm{ph}}\right)$ vs $(1 / T)$ two slopes were calculated with $E_{1}=0.7 \mathrm{eV}$ and $E_{2}=0.3 \mathrm{eV}$ (figure 3).

\section{Discussion}

The Pc single crystals were investigated after a period of time (of about five years from the time when the crystals were first grown). In the meantime they might have been affected due to exposure to ambients such as atmospheric gases (oxygen, hydrogen, etc) and the variation of humidity might have correspondingly affected the conducting mechanism. A significant amount of impurities and grain boundaries might have been present in the crystals as traps and if these impurities were present especially on the surface of the crystal the conduction mechanism would be much altered, for example, adsorbed gases and impurities might act as recombination centres (Gutmann and Lyons 1981).

The study of band gap on temperature dependence of conduction is based on the thermal excitation of electrons from the traps to the conduction band. If the traps are filled by excitation at relatively lower temperature, they may be further emptied as the temperature rises up. And the filled up traps may be emptied by the absorption of optical energy as well as by utilization of thermal energy (Bube 1960).

The observations, viz. dark activation energy (as $0.4 \mathrm{eV}$ ) and the double photo activation energies (as $0.7 \mathrm{eV}$ and $0.3 \mathrm{eV}$ ) along with the calculated band gap of $1.4 \mathrm{eV}$, could be interpreted as follows: In the dark, as the temperature increases, the electrons are excited thermally from the valence band into recombination centre $(0.3 \mathrm{eV}$ above valence band edge) and also from trapping level $(0.4 \mathrm{eV}$ below conduction band edge) into conduction band, leaving behind free holes in the valence band. In the presence of light the electrons are excited into trap level from which they are further thermally excited into conduction band.

\section{Conclusions}

Based on the observations the following band model for Pc single crystals has been suggested: The band gap of Pc single crystal is $1.4 \mathrm{eV}$ with a trap level at $0.4 \mathrm{eV}$ below the conduction band edge and a recombination level at $0.3 \mathrm{eV}$ above the valence band edge. The electrons are optically excited into the trap level initially and further they are excited thermally into the conduction band. It is assumed that the measurements have been carried out at equilibrium with a steady rate of filling and depopulation of the levels. The band model is as shown in figure 4 . The present results are compared with the results investigated earlier (Xavier and Goldsmith 1995) in table 1.

Traps are normally associated with crystal imperfections, dislocations, grain boundaries and surface of the material (Gutmann and Lyons 1981). It is very difficult to grow or synthesize very pure organic semiconductor crystals and hence the origin of 


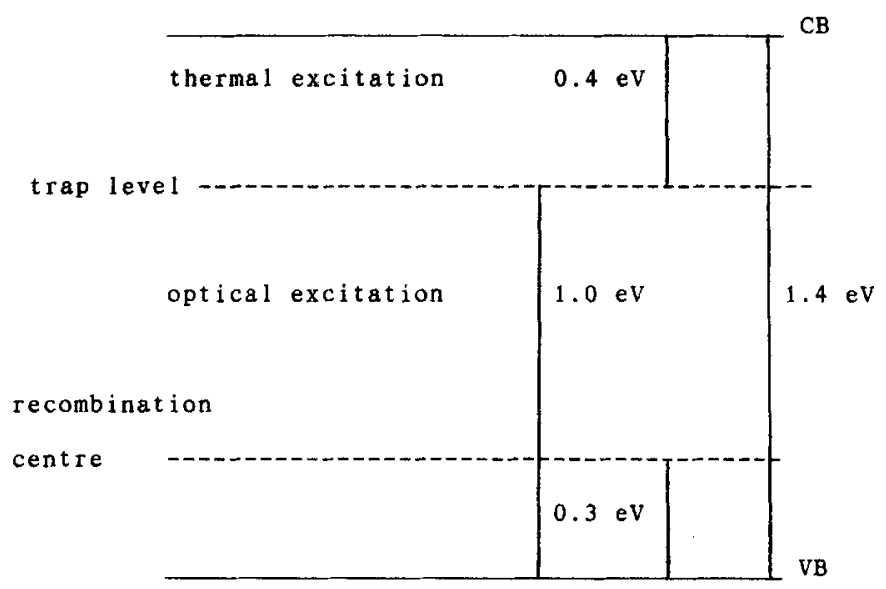

Figure 4. Band model for $\mathrm{H}_{2} \mathrm{Pc}$ single crystal.

Table 1. Band model comparison.

\begin{tabular}{|c|c|c|}
\hline & $\begin{array}{l}\text { Present } \\
\text { results }\end{array}$ & $\begin{array}{l}\text { Previous } \\
\text { results }\end{array}$ \\
\hline Dark activation energy & $0.4 \mathrm{eV}$ & $0.3 \mathrm{eV}$ \\
\hline Optical excitation energy & $1.0 \mathrm{eV}$ & $1 \cdot 14 \mathrm{eV}$ \\
\hline Recombination centre & $0 \cdot 3 \mathrm{eV}$ & $0.5 \mathrm{eV}$ \\
\hline Band gap & $1.4 \mathrm{eV}$ & $1.44 \mathrm{eV}$ \\
\hline Dark resistivity $(\mathrm{ohm} \cdot \mathrm{cm})$ & $10^{8}$ & $10^{11}$ \\
\hline Photo resistivity $(\mathrm{ohm} \cdot \mathrm{cm})$ & $0.8 \times 10^{8}$ & $2 \times 10^{9}$ \\
\hline Temperature range in dark & $305-483^{\circ} \mathrm{K}$ & $100-300^{\circ} \mathrm{K}$ \\
\hline Temperature range in light & $308-573^{\circ} \mathrm{K}$ & $100-300^{\circ} \mathrm{K}$ \\
\hline
\end{tabular}

traps could be impurities and electrical properties are shown to change due to impurity or doping (Boguslavskii and Vannikov 1970). The other source of traps could be imperfection in the crystal but in the present investigation disorder does not seem to have any appreciable effect in the experimental results, since the X-ray crystallographic diffraction study indicate no defect in crystal structure (Arulselvi and Xavier 1996). And due to weak van der Waals force inter molecular layering without creating lattice defects is possible (Akimichi et al 1993).

Due to the passage of time the crystal surface might have been affected by ambient thereby altering the sensitivity of the crystal as photoconductor. But the investigations indicate that the characteristics of the crystal are not significantly altered, e.g. though the present investigations have been carried out at higher temperature region, the temperature dependence conductivity establishes that $\mathrm{H}_{2} \mathrm{Pc}$ is thermally stable, and it indicates the same band gap model as established earlier by photoconductivity measurement technique (Xavier and Goldsmith 1995) at lower temperature range. Thus it is established that high and low temperature ranges are equivalent with respect to conduction mechanism. Over the years some changes in photo sensitivity of the crystal might be expected due to change in the ambient. But here it is seen that the ambient, especially the humidity in the air, have not had adverse effects on the crystal, thereby indicating that the crystal is also chemically stable. 


\section{Acknowledgement}

The authors express their thanks to Dr Goldsmith, Physics Department, Boston College, USA for providing the crystals used in the present investigations.

\section{References}

Akimichi H, lnoshita T, Hotta S, Noga H and Sakaki H 1993 Appl. Phys. Letts. 633158

Arulselvi A and Xavier F P 1996 Private Communication

Boer K W 1990 Survey of semiconductor physics (New York: Reinhold)

Boguslavskii L I and Vannikov A V 1970 Organic semiconductors and biopolymers (New York: Plenum)

Bube R H 1960 Photoconductivity of solids (New York: John Wiley)

Gutmann F and Lyons L E 1981 Organic semiconductors (Part A) (Malabar: Krieger)

Minami N et al 1987 Jap. J. Appl. Phys. 261754

Orti E et al 1988 J. Chem. Phys. 891009

Orti E et al 1990 J. Chem. Phys. 921228

Simon J and Andre J-J 1985 Molecular semiconductors (Berlin: Springer)

Xavier F P 1993 Optical and transport properties of phthaloc yanine and related compounds (Ann Arbor:UMI)

Xavier F P and Goldsmith G J 1995 Bull. Mater. Sci. 18269 